\title{
COLISTIN RESISTANCE ASSOCIATED WITH OUTER MEMBRANE PROTEIN CHANGE IN KLEBSIELLA PNEUMONIAE AND ENTEROBACTER ASBURIAE
}

\author{
BÉla KÁDÁR ${ }^{1 *}$, BÉla KOCSIS ${ }^{1}$, ÁKOS TÓTH ${ }^{2,3}$, KATALIN KRISTÓF ${ }^{4}$, \\ PÉTER FELSÖ $^{5}$, BÉlA KOCSIS ${ }^{5}$, KATALIN BÖDDI ${ }^{6}$ and DÓRA SZABÓ ${ }^{1}$ \\ ${ }^{1}$ Institute of Medical Microbiology, Semmelweis University, Budapest, Hungary \\ ${ }^{2} 1$ st Department of Bacteriology, National Center for Epidemiology, Budapest, Hungary \\ ${ }^{3}$ European Program for Public Health Microbiology Training (EUPHEM), European \\ Centre for Disease Prevention and Control (ECDC), Stockholm, Sweden \\ ${ }^{4}$ Diagnostic Laboratory of Clinical Microbiology, Institute of Laboratory Medicine, \\ Semmelweis University, Budapest, Hungary \\ ${ }^{5}$ Institute of Medical Microbiology and Immunology, University of Pécs, Pécs, Hungary \\ ${ }^{6}$ Institute of Biochemistry and Medical Chemistry, University of Pécs, Pécs, Hungary
}

(Received: 30 April 2017; accepted: 16 May 2017)

\begin{abstract}
In this study, outer membrane proteins (OMPs) of colistin-resistant Klebsiella pneumoniae and Enterobacter asburiae were analyzed. One colistin-susceptible and three colistin-resistant $K$. pneumoniae sequence type 258 strains as well as one colistin-susceptible E. asburiae and its colistin-heteroresistant counterpart strain were involved in the study. OMP analysis of each strain was performed by microchip method. Matrix-assisted laser desorption ionization time of flight/mass spectrometry (MALDI-TOF/MS) investigation was carried out after separation of OMPs by twodimensional gel electrophoresis and in-gel digestion. The MALDI-TOF/MS analysis of OMPs in the colistin-susceptible $K$. pneumoniae found $16 \mathrm{kDa}$ proteins belonging to the LysM domain/BON superfamily, as well as DNA starvation proteins, whereas OmpX and OmpW were detected in the colistin-resistant counterpart strains. OmpC and OmpW were detected in the colistin-susceptible E. asburiae, whereas OmpA and OmpX were identified in the colistin-resistant counterpart. This study demonstrated that OMP differences were between colistin-susceptible and -resistant counterpart strains. The altered Gram-negative cell wall may contribute to acquired colistin resistance in Enterobacteriaceae.
\end{abstract}

Keywords: MALDI-TOF/MS, outer membrane protein, colistin resistance

*Corresponding author; E-mail: kadar.bela@gmail.com 


\section{Introduction}

Klebsiella pneumoniae and Enterobacter spp. are frequently identified nosocomial Gram-negative pathogens and both are found to be resistant to multiple classes of antibiotics, including extended-spectrum cephalosporins, carbapenems, aminoglycosides, and fluoroquinolones [1-3].

The emergence of colistin resistance in K. pneumoniae was reported in several countries, and the sequence type (ST) 258 clone was most frequently identified, although strains from numerous clones (ST14, ST15, ST70, ST101, ST273, ST512, and ST1271) were also detected as colistin-resistant ones. Colistin resistance also appeared in Enterobacter spp; however, it has been detected less frequently [4-9].

The main mechanism of resistance to colistin in Gram-negative bacteria is explained by the modification of lipopolysaccharide (LPS), the target molecule of polymyxins. The addition of phosphoethanolamine and 4-deoxyaminoarabinose to the target molecule reduces its affinity to positively charged agents like polymyxins. The pmrCAB operon encodes the PmrC phosphoethanolamine transferase, the PmrA response regulator (also named as BasR), and the PmrB sensor kinase (also named as BasS), whereas the arn operon encodes the enzymes and transports proteins responsible for 4-deoxyaminoarabinose substitution [10-14].

The outer membrane bound two-component regulatory systems PmrA/ $\mathrm{PmrB}$ and PhoP/PhoQ were identified as major contributing factors in resistance to polymyxins. The insertional inactivation of the $\operatorname{mgr} B$ gene encoding another outer membrane protein (OMP) regulating $\mathrm{PhoQ} / \mathrm{PhoP}$ has also recently been associated with colistin resistance [15-17].

In Hungary, the first colistin-resistant $K$. pneumoniae strains were detected between 2008 and 2009 during an outbreak of a K. pneumoniae carbapenemase-2 (KPC-2) producing ST258 clone. Later, increasing number of acquired colistinresistant Enterobacteriaceae clinical isolates was observed. The aim of this study was to analyze one colistin-susceptible and three colistin-resistant clinical K. pneumoniae ST258 strains originating from the first Hungarian outbreak. This study also involved one colistin-susceptible Enterobacter asburiae and its colistin-heteroresistant counterpart strain. The specific aim of this study was the analysis of OMPs [18].

\section{Materials and Methods}

\section{Bacterial strains}

Three colistin-resistant and one colistin-susceptible KPC-2 producing K. pneumoniae ST258 strains were included in this study. All investigated strains 
were identified in 2008-2009 during an outbreak in Hungary. One E. asburiae strain identified from clinical urine sample and its colistin-heteroresistant counterpart were also analyzed [18].

\section{Isolation of OMPS}

The strains were inoculated into $500 \mathrm{ml}$ Mueller-Hinton Broth (Oxoid Ltd., Basingstoke, UK) and were incubated in a shaker at $37^{\circ} \mathrm{C}$ overnight. The cultures were centrifugalized $\left(6,000 \mathrm{~g}, 20 \mathrm{~min}, 4^{\circ} \mathrm{C}\right)$, then the sediments were resuspended in physiological saline solution. The suspensions were again centrifugalized and this washing step was repeated once more. The sediments were subsequently taken into $15 \mathrm{ml} 20 \mathrm{mM}$ Tris- $\mathrm{HCl}$ (pH 7.5) solution, then the bacterial cells in ice bath were disrupted with 500W ultrasound (MSE Soniprep 150 Ultrasonic Disintegrator, MSE Ltd., London, UK) twice for 2 min. Hereupon, the samples were again centrifugalized $\left(6,000 \mathrm{~g}, 20 \mathrm{~min}, 4^{\circ} \mathrm{C}\right)$, and after that, the supernatants were poured off for ultracentrifugation $\left(100,000 \mathrm{~g}, 60 \mathrm{~min}, 4^{\circ} \mathrm{C}\right)$. Thereafter, the sediments were

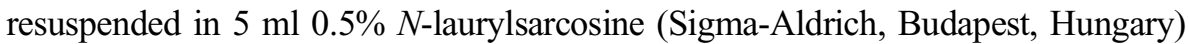
solution and incubated at room temperature for $30 \mathrm{~min}$, and then they were again ultracentrifugalized with same parameters. Finally, the sarcosine-insoluble OMPs were located in the sediment [19].

\section{OMP analysis by microchip (Agilent 2100 Bioanalyzer)}

Electrophoresis in microchips was performed in the commercially available Agilent 2100 Bioanalyzer system (Agilent Technologies, Santa Clara, CA, USA) equipped with a diode laser for fluorescence detection with 630 and $650 \mathrm{~nm}$ as excitation and emission wavelengths, respectively. OMP samples were diluted 10 times with standard labeling buffer. For the fluorescent labeling, $0.5 \mu \mathrm{L}$ of fluorescent dye/dimethyl sulfoxide solution was added to $5 \mu \mathrm{L}$ of diluted sample and incubated for $10 \mathrm{~min}$ at room temperature. The excess dye (i.e., the unbound dye) in the solutions of labeled OMPs was quenched by adding $0.5 \mu \mathrm{L}$ of ethanolamine following the reaction time. The labeled samples were diluted five times by adding $24 \mu \mathrm{L}$ of distilled water, then incubated at $100{ }^{\circ} \mathrm{C}$ for $5 \mathrm{~min}$. The samples were centrifugalized and the supernatant was used for the electrophoretic analysis, where the microchip channels were hydrodynamically filled (with pressure) with the gel matrix, the sample wells were loaded with $6 \mu \mathrm{L}$ of samples, and the respective wells were loaded with the destaining solution. The injection was made with $1,000 \mathrm{~V}$ for $80 \mathrm{~s}$ and the separation was continued at 1,000 V for $60 \mathrm{~s}$. The sample components migrated toward the anode. The temperature was maintained at $30^{\circ} \mathrm{C}$. 
Two-dimensional gel electrophoresis (2DE) of OMPs

OMPs of $K$. pneumoniae strains were separated by 2DE. A portion of $100 \mu \mathrm{g}$ of the total protein content of the OMPs were supplemented with 2DE sample buffer $8 \mathrm{M}$ urea (Bio-Rad, Budapest, Hungary), 2\% CHAPS (Bio-Rad), $50 \mathrm{mM}$ dithiothreitol (DTT) (Bio-Rad), 0.2\% Biolyte 3/10 ampholytes (Bio-Rad), and a trace of bromophenol blue (Bio-Rad) to a total volume of $125 \mu \mathrm{l}$, then the immobilized $\mathrm{pH}$ gradient strips of length $7 \mathrm{~cm}, \mathrm{pH} \mathrm{3-10,} \mathrm{(Bio-Rad)} \mathrm{were}$ incubated for rehydration overnight. Isoelectric focusing (IEF) of the OMPs was performed on an IEF cell (Bio-Rad) using the following program: $250 \mathrm{~V}, 2 \mathrm{~h}$, linear, $500 \mathrm{~V}, 2 \mathrm{~h}$, linear, 4,000 V, 10,000 Vh, rapid. After the IEF, the strips were equilibrated twice for $10 \mathrm{~min}$ in equilibration buffer containing $6 \mathrm{M}$ urea, $2 \%$ sodium dodecyl sulfate (SDS), 20\% glycerol, a trace of bromophenol blue, and 2\% DTT (Bio-Rad). During the second equilibration step, 2.5\% IAA was used instead of DTT. After the equilibration, the strips were applied to the second dimension [12\% sodium dodecyl sulfate polyacrylamide gel electrophoresis (SDS-PAGE), $8 \times 6 \mathrm{~cm}$ (Bio-Rad)]. The separation of the proteins according to their masses was performed at $80 \mathrm{~V}$ for $20 \mathrm{~min}$ and $120 \mathrm{~V}$ until the end of the run. After the SDSPAGE, the gels were stained with Coomassie blue R-250. Protein marker (ladder, Bio-Rad Precision Plus Protein ${ }^{\mathrm{TM}}$ Kaleidoscope $^{\mathrm{TM}}$, Bio-Rad) was used as a molecular standard. The gels were scanned on Pharos FX laser scanner (BioRad). For the identification of the OMPs and the mass spectrometric analysis, the spots of interest were excised from the gels.

\section{In-gel digestion}

Protein bands were excised from gels and were cut to small pieces and digested using modified version of the protocol developed by Shevchenko et al. [20]. Coomassie blue and SDS were removed with $100 \mathrm{mM}$ ammonium bicarbonate (Bio-Rad), then the gel slabs were dehydrated with acetonitrile (ACN). Disulfide bridges were reduced with $10 \mathrm{mM}$ DTT (Bio-Rad), then the free sulfhydryl ( $\mathrm{SH})$ groups were alkylated with $55 \mathrm{mM}$ iodacetamide solution (Bio-Rad). The modified proteins were in-gel digested with side-chain-protected trypsin (Promega, Madison, WI, USA) in $50 \mathrm{mM}$ ammonium bicarbonate overnight at $37^{\circ} \mathrm{C}$. The digested peptides were extracted from the gel with $5 \%$ formic acid solution (Sigma-Aldrich) in a 2:1 mixture of ACN (SigmaAldrich) and water. The extracted peptides were evaporated to dryness and redissolved in $5 \mu \mathrm{L}$ of $0.1 \%$ trifluoroacetic acid (TFA) in water before the mass spectrometric measurement $[20,21]$. 
Matrix-assisted laser desorption ionization time of flight/mass spectrometry (MALDI-TOF/MS)

Mass analysis was performed on an Autoflex II MALDI-TOF/MS instrument (Bruker Daltonics, Bremen, Germany). During MS analysis of the digested proteins, $8 \mathrm{mg}$ of $\alpha$-cyano-4-hydroxycinnamic acid (Bruker Daltonics, Bremen, Germany) was dissolved in $1 \mathrm{~mL}$ of $50 \% \mathrm{ACN}$ and $0.1 \%$ TFA (Scharlau Chemie, Barcelona, Spain) in water. In each case, $1 \mu \mathrm{L}$ of the matrix was deposited on a stainless steel target together with $1 \mu \mathrm{L}$ of the sample with dried droplet method.

All mass spectra were monitored in positive mode with pulsed ionization [l=337 nm; nitrogen laser (MNL $106 \mathrm{PD})]$ with a maximum pulse rate of $50 \mathrm{~Hz}$. Peptides of the digests were measured in positive reflectron mode using a delayed extraction of $120 \mathrm{~ns}$. The accelerating voltage was set to $+19 \mathrm{kV}$ and the reflectron voltage was set to $+20 \mathrm{kV}$. The spectra of peptides were the sum of 1,000 shots; external calibration has been implemented. Data processing was executed with flexAnalysis software packages (version: 3.1; Bruker Daltonics, Bremen, Germany). Sequence Editor software (Bruker Daltonics) was used for the analysis with the following criteria: (i) all cysteines were supposed to be treated with iodoacetamide, (ii) monoisotopic masses were allowed, and (iii) the maximum number of missed cleavage sites was two [22,23]. Data analysis was performed and protein identification was achieved using the MASCOT algorithm (http:// www.matrixscience.com) and the Swiss-Prot entries database (Swiss Institute of Bioinformatics, Geneva, Switzerland), where (i) carbamidomethyl cysteine as fixed modification and (ii) oxidation of methionine were allowed as variable modifications. Mass accuracy was considered within $150 \mathrm{ppm}$ for MS and $0.8 \mathrm{Da}$ for MS/MS. Only proteins with at least two matching sequences were considered $[20,24]$.

\section{Results and Discussion}

Our investigations regarding the colistin resistance mechanisms found OMP change in the colistin-resistant strains compared with the colistin-susceptible ones. The Agilent 2100 Bioanalyzer showed a distinct lack of protein fraction in the colistin-resistant $K$. pneumoniae and $E$. asburiae isolates during the runs of OMPs, whereas the same fraction was present in the colistin-susceptible ones (Figures 1 and 2).

Various OMPs were detected by MALDI-TOF/MS after 2DE separation of OMPs (Table I). In the colistin-susceptible $K$. pneumoniae strain, 15-16 kDa 


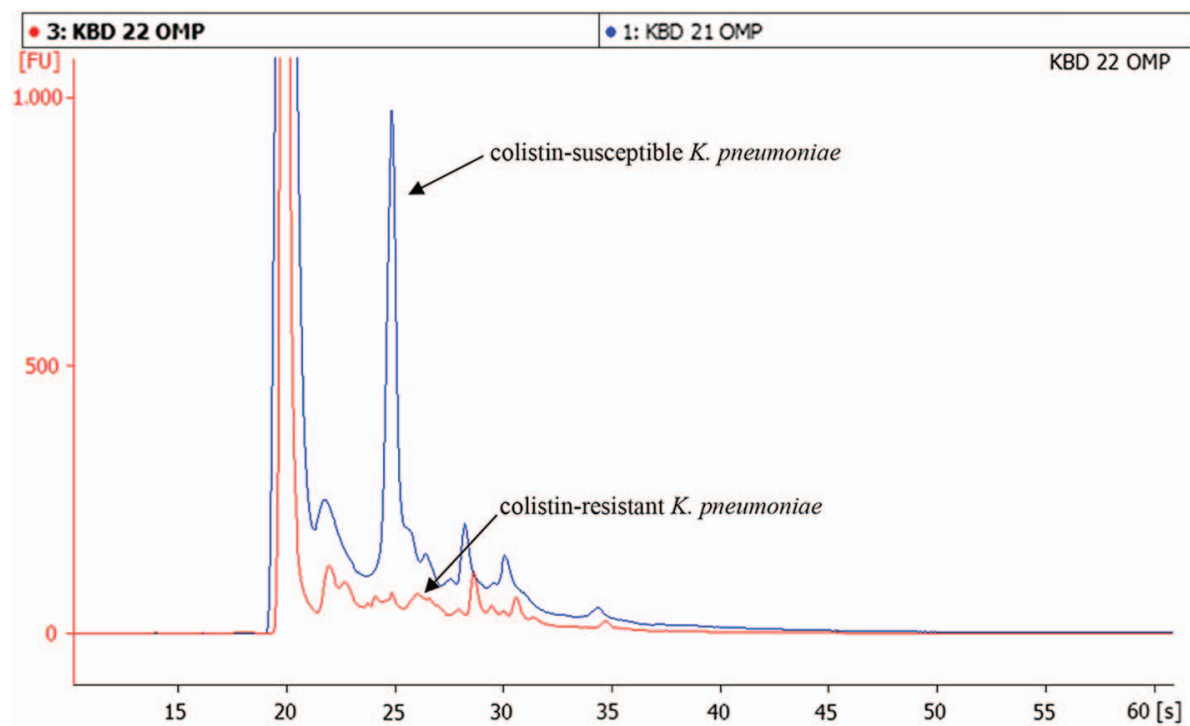

Figure 1. OMP analysis of colistin-susceptible and colistin-resistant K. pneumoniae strains (colistinsusceptible strain: blue line; colistin-resistant strain: red line)

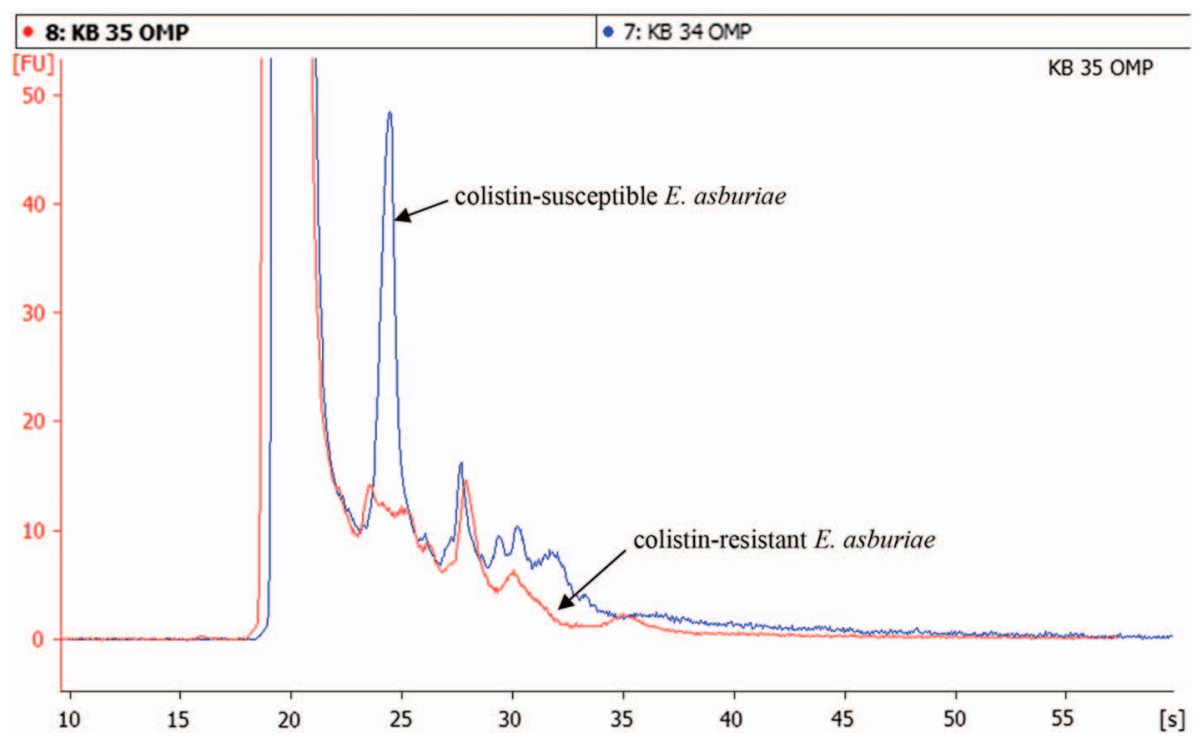

Figure 2. OMP analysis of colistin-susceptible E. asburiae strain and its heteroresistant counterpart (colistin-susceptible strain: blue line; colistin-resistant strain: red line) 
Table I. OMPs detected in colistin-susceptible and colistin-resistant K. pneumoniae and E. asburiae

\begin{tabular}{lllc}
\hline Strain & \multicolumn{1}{c}{ Protein } & Protein ID & Molecular weight (Da) \\
\hline col S Kpn 11 & LysM BON superfamily & gi|152970277 & 15,899 \\
& DNA starvation protein & gi|152969398 & 18,697 \\
col R Kpn 12 & OmpW & gi|152969802 & 24,321 \\
col R Kpn 97 & OmpW, colicin S4 rec; & A6T7W0 & 24,321 \\
& putative transport protein & & \\
& OmpX & S5YR85 & 14,866 \\
col R Kpn 105 & OmpX & F0JWH6 & 18,680 \\
& OmpW & G0GLV0 & 22,969 \\
col S Easb & OmpC & K4YC30 & 40,459 \\
& OmpW & G0GLV0 & 22,969 \\
col R Easb & flagellin & V3HNK5 & 28,529 \\
& OmpA & V3PJ21 & 37,748 \\
& OmpX & V3E259 & 18,654 \\
\hline
\end{tabular}

Note: col S Kpn: colistin-susceptible K. pneumoniae; col R Kpn: colistin-resistant K. pneumoniae; col S Easb: colistin-susceptible E. asburiae; col R Easb: colistin-resistant E. asburiae.

proteins of the LysM domain /BON superfamily and DNA starvation/stationary phase protection proteins were detected, whereas in the colistin-resistant counterpart strain, these proteins were absent, but OmpX (19 kDa) and OmpW (24 kDa) were present. In the colistin-susceptible E. asburiae, OmpC (40 kDa) and OmpW $(23 \mathrm{kDa})$ were identified, whereas in the colistin-heteroresistant counterpart strain, OmpA $(38 \mathrm{kDa})$ and OmpX $(19 \mathrm{kDa})$ were detected.

DNA starvation proteins (Dps) were found in several bacteria, their main function being the protection of bacteria during the stationary phase of cell division. They bind to the bacterial chromosome in a non-specific way creating a stable, condensed DNA starvation/stationary phase protection protein (DpsDNA) complex which is protected against harmful effects. Furthermore, they bind intracellular $\mathrm{Fe}^{2+}$ ions and oxidize them into $\mathrm{Fe}^{3+}$ ions, then store them indirectly decreasing the amount of intracellular reactive oxygen species $[25,26]$.

LysM and BON domains are the conserved sections found in many bacterial proteins. These are mainly structural proteins and enzymes responsible for maintaining cell membrane integrity, and they are present in the greatest amount during the stationary cell division phase. The LysM domain specifically binds to the $\mathrm{N}$-acetylglucosamine molecules of peptidoglycan. As colistin resistance is based on molecular changes in the cell wall, the alterations in the expression of these structural proteins may contribute to its development [22, 27].

OmpC and its homologues (Omp36 and OmpK36) are porin-type transport proteins found in the outer membrane of Enterobacteriaceae. They are responsible for transporting several types of molecules into the cells, including antibiotics 
(e.g., cephalosporins, carbapenems, and fluoroquinolones). Their loss or decreased expression leads to antibiotic resistance and diminished susceptibility to serum antimicrobial activity in Escherichia coli and Enterobacter spp. [23, 28].

OmpA is a multifunctional membrane protein: in addition to maintaining integrity of the outer membrane, it is responsible for serum resistance in E. coli and antimicrobial peptide resistance in $K$. pneumoniae [29, 30].

OmpX is a protein structurally similar to OmpA. Its overproduction was observed in multiresistant Enterobacter aerogenes strains with the simultaneous underproduction of OmpF and Omp36 porins, as well as structural changes of LPS. Upregulation of ompX and downregulation of omp36 together cause the decrease of outer membrane permeability [31-33].

The reports of OmpA and OmpX contributing to antimicrobial peptide resistance and outer membrane structural changes in Enterobacteriaceae and underproduction of OmpC leading to serum resistance in Enterobacter spp. concur with our previous observation of colistin-resistant E. asburiae and $K$. pneumoniae strains being tolerant to antimicrobial peptides [34].

This study identified OMP change as a possible mechanism for colistin resistance in K. pneumoniae ST258 and E. asburiae. Protein assortment change in the outer membrane may contribute to the stability and integrity of cell wall, thus developing and maintaining colistin resistance. Further investigations are required to explore the potentially elaborate regulatory system and the exact roles of the different proteins.

\section{Acknowledgements}

This study was financially supported by the Hungarian Research Fund, Grant Number: OTKA K 108481. Investigations of MALDI-TOF/MS and 2D electrophoresis were financially supported by PTE ÁOK/KA-2013/5 KA research grant.

\section{Conflict of Interest}

The authors declare no conflict of interest.

\section{References}

1. Paterson, D. L.: Resistance in Gram-negative bacteria: Enterobacteriaceae. Am J Infect Control 34, S20-S28; discussion S64-S73 (2006).

2. Delgado-Valverde, M., Sojo-Dorado, J., Pascual, A., Rodríguez-Baño, J.: Clinical management of infections caused by multidrug-resistant Enterobacteriaceae. Ther Adv Infect Dis 1, 49-69 (2013). 
3. Bassetti, M., Peghin, M., Pecori, D.: The management of multidrug-resistant Enterobacteriaceae. Curr Opin Infect Dis 29, 583-594 (2016).

4. Antoniadou, A., Kontopidou, F., Poulakou, G., Koratzanis, E., Galani, I., Papadomichelakis, E., Kopterides, P., Souli, M., Armaganidis, A., Giamarellou, H.: Colistin-resistant isolates of Klebsiella pneumoniae emerging in intensive care unit patients: First report of a multiclonal cluster. J Antimicrob Chemother 59, 786-790 (2007).

5. Marchaim, D., Chopra, T., Pogue, J. M., Perez, F., Hujer, A. M., Rudin, S., Endimiani, A., Navon-Venezia, S., Hothi, J., Slim, J., Blunden, C., Shango, M., Lephart, P. R., Salimnia, H., Reid, D., Moshos, J., Hafeez, W., Bheemreddy, S., Chen, T. Y., Dhar, S., Bonomo, R. A., Kaye, K. S.: Outbreak of colistin-resistant, carbapenem-resistant Klebsiella pneumoniae in metropolitan Detroit, Michigan. Antimicrob Agents Chemother 55, 593-599 (2011).

6. Bogdanovich, T., Adams-Haduch, J. M., Tian, G. B., Nguyen, M. H., Kwak, E. J., Muto, C. A., Doi, Y.: Colistin-resistant, Klebsiella pneumoniae carbapenemase (KPC)-producing Klebsiella pneumoniae belonging to the international epidemic clone ST258. Clin Infect Dis 53, 373-376 (2011).

7. Mammina, C., Bonura, C., Di Bernardo, F., Aleo, A., Fasciana, T., Sodano, C., Saporito, M. A., Verde, M. S., Tetamo, R., Palma, D. M.: Ongoing spread of colistin-resistant Klebsiella pneumoniae in different wards of an acute general hospital, Italy, June to December 2011. Euro Surveill 17, 20248 (2012).

8. Boo, T. W., O’Connell, N., Power, L., O’Connor, M., King, J., McGrath, E., Hill, R., Hopkins, K. L., Woodford, N.: First report of IMI-1-producing colistin-resistant Enterobacter clinical isolate in Ireland, March 2013. Euro Surveill 18, 20548 (2013).

9. Huang, L., Wang, X., Feng, Y., Xie, Y., Xie, L., Zong, Z.: First identification of an IMI-1 carbapenemase-producing colistin-resistant Enterobacter cloacae in China. Ann Clin Microbiol Antimicrob 14, 51 (2015).

10. Gunn, J. S., Miller, S. I.: PhoP-PhoQ activates transcription of pmrAB, encoding a twocomponent regulatory system involved in Salmonella typhimurium antimicrobial peptide resistance. J Bacteriol 178, 6857-6864 (1996).

11. Breazeale, S. D., Ribeiro, A. A., McClerren, A. L., Raetz, C. R.: A formyltransferase required for polymyxin resistance in Escherichia coli and the modification of lipid A with 4-amino-4-deoxy-L-arabinose. Identification and function of UDP-4-deoxy-4-formamidoL-arabinose. J Biol Chem 280, 14154-14167 (2005).

12. Kline, T., Trent, M. S., Stead, C. M., Lee, M. S., Sousa, M. C., Felise, H. B., Nguyen, H. V., Miller, S. I.: Synthesis of and evaluation of lipid A modification by 4-substituted 4-deoxy arabinose analogs as potential inhibitors of bacterial polymyxin resistance. Bioorg Med Chem Lett 18, 1507-1510 (2008).

13. Gunn, J. S.: The Salmonella PmrAB regulon: Lipopolysaccharide modifications, antimicrobial peptide resistance and more. Trends Microbiol 16, 284-290 (2008).

14. Falagas, M. E., Rafailidis, P. I., Matthaiou, D. K.: Resistance to polymyxins: Mechanisms, frequency and treatment options. Drug Resist Updat 13, 132-138 (2010).

15. Cheng, H. Y., Chen, Y. F., Peng, H. L.: Molecular characterization of the PhoPQ-PmrDPmrAB mediated pathway regulating polymyxin B resistance in Klebsiella pneumoniae CG43. J Biomed Sci 17, 60 (2010).

16. Cannatelli, A., D’Andrea, M. M., Giani, T., Di Pilato, V., Arena, F., Ambretti, S., Gaibani, P., Rossolini, G. M.: In vivo emergence of colistin resistance in Klebsiella pneumoniae 
producing KPC-type carbapenemases mediated by insertional inactivation of the $\mathrm{PhoQ} /$ PhoP $m g r B$ regulator. Antimicrob Agents Chemother 57, 5521-5526 (2013).

17. Kocsis, B., Kádár, B., Tóth, Á., Fullár, A., Szabó, D.: MgrB variants in colistinsusceptible and colistin-resistant Klebsiella pneumoniae ST258. J Microbiol Immunol Infect (2016). doi:10.1016/j.jmii.2016.06.008 [Epub Ahead of Print].

18. Tóth, Á., Damjanova, I., Puskás, E., Jánvári, L., Farkas, M., Dobák, A., Böröcz, K., Pászti, J.: Emergence of a colistin-resistant KPC-2-producing Klebsiella pneumoniae ST258 clone in Hungary. Eur J Clin Microbiol Infect Dis 29, 765-769 (2010).

19. Baik, S. C., Kim, K. M., Song, S. M., Kim, D. S., Jun, J. S., Lee, S. G., Song, J. Y., Park, J. U., Kang, H. L., Lee, W. K., Cho, M. J., Youn, H. S., Ko, G. H., Rhee, K. H.: Proteomic analysis of the sarcosine-insoluble outer membrane fraction of Helicobacter pylori strain 26695. J Bacteriol 186, 949-955 (2004).

20. Shevchenko, A., Tomas, H., Havlis, J., Olsen, J. V., Mann, M.: In-gel digestion for mass spectrometric characterization of proteins and proteomes. Nat Protoc 1, 2856-2860 (2006).

21. Markó, L., Szigeti, N., Szabó, Z., Böddi, K., Takátsy, A., Ludány, A., Kõszegi, T., Molnár, G. A., Wittmann, I.: Potential urinary biomarkers of disease activity in Crohn's disease. Scand J Gastroenterol 45, 1440-1448 (2010).

22. Yeats, C., Bateman, A.: The BON domain: A putative membrane-binding domain. Trends Biochem Sci 28, 352-355 (2003).

23. Yigit, H., Anderson, G. J., Biddle, J. W., Steward, C. D., Rasheed, J. K., Valera, L. L., McGowan, J. E., Jr., Tenover, F. C.: Carbapenem resistance in a clinical isolate of Enterobacter aerogenes is associated with decreased expression of $\mathrm{OmpF}$ and $\mathrm{OmpC}$ porin analogs. Antimicrob Agents Chemother 46, 3817-3822 (2002).

24. Böddi, K., Takátsy, A., Szabó, S., Markó, L., Márk, L., Wittmann, I., Ohmacht, R., Montskó, G., Vallant, R. M., Ringer, T., Bakry, R., Huck, C. W., Bonn, G. K., Szabó, Z.: Use of fullerene-, octadecyl-, and triaconthyl silica for solid phase extraction of tryptic peptides obtained from unmodified and in vitro glycated human serum albumin and fibrinogen. J Sep Sci 32, 295-308 (2009).

25. Zhao, G., Ceci, P., Ilari, A., Giangiacomo, L., Laue, T. M., Chiancone, E., Chasteen, N. D.: Iron and hydrogen peroxide detoxification properties of DNA-binding protein from starved cells. A ferritin-like DNA-binding protein of Escherichia coli. J Biol Chem 277, 2768927696 (2002).

26. Karas, V. O., Westerlaken, I., Meyer, A. S.: The DNA-binding protein from starved cells (Dps) utilizes dual functions to defend cells against multiple stresses. J Bacteriol 197, 32063215 (2015).

27. Visweswaran, G. R., Leenhouts, K., van Roosmalen, M., Kok, J., Buist, G.: Exploiting the peptidoglycan-binding motif, LysM, for medical and industrial applications. Appl Microbiol Biotechnol 98, 4331-4345 (2014).

28. Liu, Y. F., Yan, J. J., Lei, H. Y., Teng, C. H., Wang, M. C., Tseng, C. C., Wu, J. J.: Loss of outer membrane protein $\mathrm{C}$ in Escherichia coli contributes to both antibiotic resistance and escaping antibody-dependent bactericidal activity. Infect Immun 80, 1815-1822 (2012).

29. Wang, Y.: The function of OmpA in Escherichia coli. Biochem Biophys Res Commun 292, 396-401 (2002).

30. Llobet, E., March, C., Giménez, P., Bengoechea, J. A.: Klebsiella pneumoniae OmpA confers resistance to antimicrobial peptides. Antimicrob Agents Chemother 53, 298-302 (2009). 
31. Stoorvogel, J., van Bussel, M. J., van de Klundert, J. A.: Biological characterization of an Enterobacter cloacae outer membrane protein (OmpX). J Bacteriol 173, 161-167 (1991).

32. Gayet, S., Chollet, R., Molle, G., Pagès, J. M., Chevalier, J.: Modification of outer membrane protein profile and evidence suggesting an active drug pump in Enterobacter aerogenes clinical strains. Antimicrob Agents Chemother 47, 1555-1559 (2003).

33. Dupont, M., Dé, E., Chollet, R., Chevalier, J., Pagès, J. M.: Enterobacter aerogenes OmpX, a cation-selective channel mar- and osmo-regulated. FEBS Lett 569, 27-30 (2004).

34. Kádár, B., Kocsis, B., Kristóf, K., Tóth, Á., Szabó, D.: Effect of antimicrobial peptides on colistin-susceptible and colistin-resistant strains of Klebsiella pneumoniae and Enterobacter asburiae. Acta Microbiol Immunol Hung 62, 501-508 (2015). 